\title{
Ablation and Plasma Formation Due to Laser Irradiance
}

\author{
Saravanakanthan Rajendran ${ }^{1}$, Michael Keidar ${ }^{2}$ and Iain D. Boyd ${ }^{3}$ \\ University of Michigan, Ann Arbor, MI 48109, USA
}

\begin{abstract}
A numerical model has been developed to describe the laser induced ablation of metal surfaces and the subsequent expansion of plasma above the surface. The model describes the absorption of the laser energy by the metal and the resulting temperature rise in the surface which then induces ablation of the target material. The ejection of material from the surface forms a plasma in the vicinity of the irradiated material that expands into the ambient vacuum and also absorbs the laser irradiation passing through it. Results for an aluminum target irradiated with a $\mathrm{KrF}$ laser were obtained. Temperature profiles in the target material, the ablation rate and the plasma expansion results are presented here. Research shows that laser absorption by the plasma becomes significant at higher fluences, which significantly affects target heating as well as plasma expansion. The results presented here are for cases when absorption by the plasma is not very significant when the plasma is still transparent to the laser radiation. The results show acceptable agreement with other analytical models.
\end{abstract}

\section{Introduction}

$\mathrm{T}$ HE incidence of a high-power laser pulse onto a metal target leads to local heating and subsequent evaporation of the material. The ablated material expands and forms a plume front in the ambient atmosphere, which in this case is assumed to initially be a vacuum. If the ablation were to occur in ambient air ${ }^{1}$, within the plasma and near the shock front created by the material ejection, the high temperatures causes complex chemical reactions leading to ionization, recombination, and excitation. In addition, chemical reactions between the ablated material and air molecules are possible at these elevated air temperatures.

A number of numerical models have been proposed to analyze the laser-solid interaction and ablation process $^{11,12,14,15}$. We developed a model based on a kinetic description of the Knudsen layer and a hydrodynamic description of the collision-dominated plasma region. Preliminary analysis of the laser ablation of different targets subjected to directed-energy impact was performed. The ablation rate was found to depend on the surface temperature as well as plasma density and temperature in the target vicinity ${ }^{2}$. In this present paper, we report on an initial attempt to integrate this ablation model to a self-consistent analytical model to simulate the laser-target interaction during directed energy impact. The complete model couples analysis of target heating and evaporation to plume formation and expansion. The absorption of laser beam energy by the plume represents an important coupling back to both plume expansion and laser-target interaction. Therefore the different components of the model are solved simultaneously as a function of time in order to produce an accurate description of the laser ablation mechanism, plume expansion and plasma formation.

We consider the general case of an aluminum target being irradiated by a $\mathrm{KrF}$ laser in vacuum conditions. The model predicts the ablation rate and the depth of the resulting crater, as well as the temperature profile in the target material. Furthermore, the temporal behavior of the expanding plume is also obtained.

\section{Model}

\section{A. Laser Beam}

The laser pulse assumed in this study had a Gaussian profile with FWHM of 8 ns, and centered at 15 ns. Furthermore, a $\mathrm{KrF}$ laser was modeled, with wavelength, $\lambda$, of $248 \mathrm{~nm}$. In this study, the laser fluence is chosen as the parameter, and the laser beam intensity is calculated to produce the required fluence. Laser fluences in the range

\footnotetext{
${ }^{1}$ Graduate Student Research Assistant, Department of Aerospace Engineering, AIAA Student Member.

${ }^{2}$ Assistant Research Scientist, Department of Aerospace Engineering, AIAA Senior Member.

${ }^{3}$ Professor, Department of Aerospace Engineering, AIAA Associate Fellow.
} 
of $1.0-2.0 \mathrm{~J} / \mathrm{cm}^{2}$ are considered in this study, corresponding to laser irradiances in the range of $10^{8} \mathrm{~W} / \mathrm{cm}^{2}$. An example of the laser beam profile is shown in Fig. 1 for the case of $2.0 \mathrm{~J} / \mathrm{cm}^{2}$.

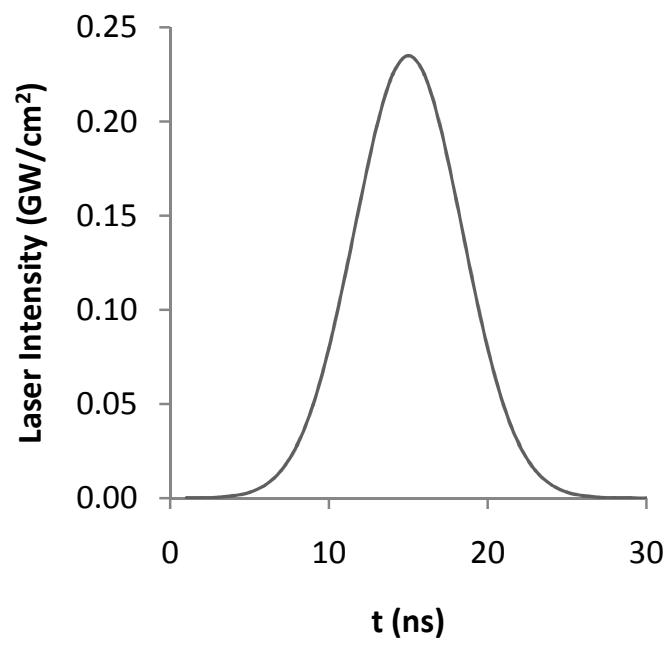

Figure 1. Laser beam intensity profile assumed in the model.

\section{B. Surface Temperature}

A macroscopic description of the laser-solid interaction is used in this study, whereby the thermal heat conduction equation is used to analyze heating of the metal. This approach is valid for laser interaction with metals, in which case laser light is absorbed by interaction with electrons, and is then transferred to lattice phonons by collisions. For metals, the energy relaxation time is of the order of $10^{-13} \mathrm{~s}$, and therefore for ns-pulsed laser beams we can assume optical energy is turned instantaneously into heat, allowing the application of the heat conduction equation ${ }^{3}$.

The temperature rise in the target material due to the laser beam will be computed from the following equation ${ }^{4}$,

$$
c \rho \frac{\partial T(z, t)}{\partial t}=\nabla\{K(T(z, t)) \nabla T(z, t)\}+q_{\text {laser }}(z, t)
$$

where $c$ is the specific heat of the material, $\rho$ is the material density, $K$ is the temperature dependent thermal conductivity and $q_{\text {laser }}$ is the heat source due to laser radiation.

The direction into the target is taken as the positive-z axis. The first term on the right-hand side represents the heat conduction in the metal. The heat source term in Eq. (1), which is due to the absorption of laser beam radiation, is given by

$$
q_{\text {laser }}(z, t)=\mu(z) I(z, t)
$$

where $\mu$ is the absorption coefficient and I is the laser beam irradiance as a function of time and position in the target. The laser beam will be absorbed by the metal as it travels through the target material, causing its intensity to drop. This change in the laser beam intensity is given by the Beer-Lambert law ${ }^{4}$,

$$
I(z, t)=A I_{o}(t) \exp \left[-\int_{0}^{z} \mu(z, t) d z\right]
$$

where $A$ is the surface absorptivity and $I_{0}$ is the irradiance of the incident beam. The surface absorptivity is defined as $(1-R)$ where $R$ is the surface reflectivity, and represents the fraction of laser energy that is reflected by the surface. Therefore $A$ represents the fraction of energy that is absorbed by the material. It is assumed that the deposited part of the laser energy is instantaneously converted into heat. The effect of evaporation of the metal surface is incorporated 
into the model in order to obtain an accurate description of the thermal field in the target material. Convection and radiation effects will be included in the future to better describe the heating effect of the laser.

\section{Plasma Density}

The plasma density at the surface can be determined if the equilibrium vapor pressure can be specified. The following relation ${ }^{5}$ gives the equilibrium vapor pressure at the surface for aluminum as

$$
\log _{10} p_{0}=11.60-15880 / T_{0}
$$

where $T_{0}$ is the surface temperature. The equation of state, $p_{0}=n_{0} k T_{0}$, can then be used to determine the density at the surface.

\section{Ablation Rate}

The ablation rate will be calculated using the kinetic model described in Keidar et $\mathrm{al}^{6}$. A schematic representation of the plasma and kinetic layers near the surface of the target is shown in Fig. 2. In the kinetic region, using Anisimov's assumption ${ }^{7}$ that the velocity distribution function for the returned particles is $\beta f_{1}(V)$, where $\beta$ is a proportionality coefficient, the relation of the heavy particle parameters at the outer boundary of the kinetic layer is given by the following set of equations

$$
\begin{gathered}
\frac{n_{0}}{2\left(\pi d_{0}\right)^{0.5}}=n_{1} V_{1}+\beta \frac{n_{1}}{2\left(\pi d_{1}\right)^{0.5}} \times\left\{\exp \left(-\alpha^{2}\right)-\alpha \pi^{0.5} \operatorname{erfc}(\alpha)\right\} \\
\frac{n_{0}}{4 d_{0}}=\frac{n_{1}}{2 d_{1}}\left\{\left(1+2 \alpha^{2}\right)-\beta\left[\left(0.5+\alpha^{2}\right) \operatorname{erfc}(\alpha)-\alpha \exp \left(-\alpha^{2}\right) / \pi^{0.5}\right]\right\} \\
\frac{n_{0}}{\left(\pi d_{0}\right)^{1.5}}=\frac{n_{1}}{\pi d_{1}^{1.5}}\left\{\alpha\left(\alpha^{2}+2.5\right)-0.5 \beta\left[\alpha\left(\alpha^{2}+2.5\right) \operatorname{erfc}(\alpha)-\left(\alpha^{2}+2\right) \exp \left(-\alpha^{2}\right) / \pi^{0.5}\right]\right\}
\end{gathered}
$$

where $\alpha=V_{1} /\left(2 k T_{1} / m\right)^{0.5}, d_{0}=m /\left(2 k T_{0}\right), d_{1}=m /\left(2 k T_{1}\right), \operatorname{erfc}(\alpha)=1-\operatorname{erf}(\alpha), \operatorname{erf}(\alpha)=$ error function.

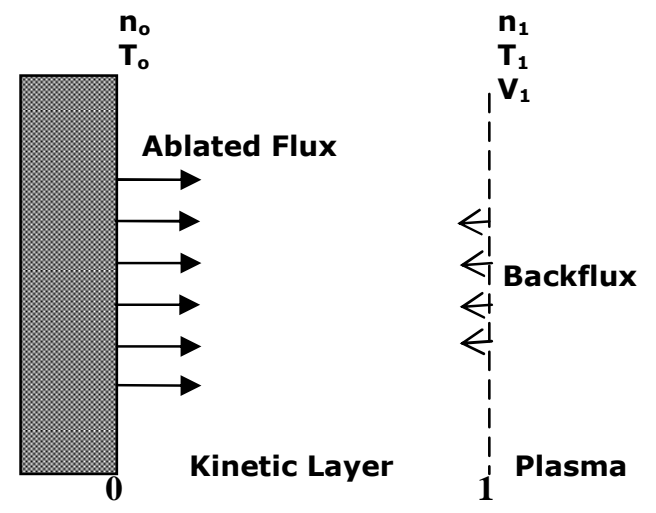

Figure 2. Schematic representation of the kinetic layer near the surface.

For relatively small plasma densities, we can assume that the plasma accelerates to the sound speed at the edge of the kinetic layer ${ }^{6}$, and in this case the solution of the kinetic layer Eqs.(5) gives the plasma density at the edge of this layer as being equal to $0.34 n_{0}$ and the temperature as $0.67 T_{0}$. Another approach is to solve Eqs. (5) along with mass and momentum conservation relations between the edge of the kinetic layer and the plasma bulk ${ }^{2}$. Using this approach, the velocity ratio $\alpha$ is calculated as part of the solution. The ablation rate is then given by the relation ${ }^{13}$

$$
G=m n_{1} V_{1}
$$


where $m$ is the mass of aluminum particles in $\mathrm{kg} ., n_{l}$ and $V_{l}$ are the density and velocity at the edge of the kinetic layer respectively. If we assume expansion to sound speed, the velocity at the edge of the kinetic layer is then given by

$$
V_{1}=\sqrt{2 k T_{1} / m}
$$

\section{E. Plasma Expansion}

The heating of the target and subsequent ablation of material forms a plume in the vacuum above the surface that continues to develop with increasing temperature and density, causing the ionization of the vapor and the formation of a plasma. This rise in temperature and density means the collisions between particles becomes frequent enough for the assumption of local thermodynamic equilibrium to be adopted for the description of the plume expansion. This assumption implies that thermal equilibrium is established between neutrals, ions and electrons in a sufficiently small region of the vapor and a common temperature can be used to characterize them. Furthermore, the SahaEggert equation ${ }^{1}$ can then be utilized to determine the fraction of electrons, ions and neutrals in the vapor. Assuming a quasi-neutral single element plasma and neglecting the difference between the partition function of ions and neutrals, the Saha-Eggert equation for each ionization step can be written as

$$
\frac{x_{e} x_{z}}{x_{z-1}}=\frac{1}{n}\left(\frac{2 \pi m_{e} k T}{h^{2}}\right)^{3 / 2} \exp \left(-\frac{E_{z}}{k T}\right)
$$

where $h$ stands for the Planck constant, $k$ is the Boltmann constant and $E_{z}$ is the ionization potential of species with charge number $z$. The local number density, $n$, is given by $n=\rho / m$; where $\rho$ is the mass density of the plasma and $m_{e}$ is the electron mass. The ionization degrees, $x_{e}$ and $x_{z}$, which represent the fraction of electron and ions of charge $z$ are defined as $x_{e}=n_{e} / n$ and $x_{z}=n_{z} / n$ respectively. In this study, since aluminum was used as the target material, three different Saha-Eggert equations are incorporated in the model to obtain the composition of $\mathrm{Al}^{+}, \mathrm{Al}^{2+}$ and $\mathrm{Al}^{3+}$ ions in the plasma.

The Saha-Eggert equations for each ionization step are supplemented by two equations for the conservation of matter and charge to obtain a complete set of equations, which for this study were

$$
\begin{aligned}
& x_{0}+x_{1}+x_{2}+x_{3}=0 \\
& x_{1}+2 x_{2}+3 x_{3}=x_{e}
\end{aligned}
$$

The Newton-Raphson iterative method ${ }^{17}$ is used to solve this strongly non-linear system of equations in order to obtain relatively fast and reliable convergence.

The expansion of the evaporated material in the vicinity of the ablated surface is modeled using the onedimensional Euler equations. These hydrodynamic equations express the conservation of mass, momentum and energy, and in this case have an energy source term to account for the absorption of laser irradiation by the plasma ${ }^{18}$

$$
\begin{gathered}
\frac{\partial \rho}{\partial t}=-\frac{\partial(\rho v)}{\partial x} \\
\frac{\partial(\rho v)}{\partial t}=-\frac{\partial}{\partial x}\left[p+\rho v^{2}\right] \\
\frac{\partial}{\partial t}\left[\rho\left(e+\frac{v^{2}}{2}\right)\right]=-\frac{\partial}{\partial x}\left[\rho v\left(e+\frac{p}{\rho}+\frac{v^{2}}{2}\right)\right]+\alpha_{I B} \Phi-\varepsilon_{r a d}
\end{gathered}
$$

where $\rho$ represents the local mass density, $p$ denotes the local pressure, $v$ is the local vapor velocity, and $\rho e$ is the local internal energy density. Assuming that the evaporated material follows the ideal gas law, the pressure and internal energy density are given by ${ }^{18}$

$$
\begin{gathered}
p=\left(1+x_{e}\right) \frac{\rho k T}{m} \\
\rho e=\frac{\rho}{m}\left[\frac{3}{2}\left(1+x_{e}\right) k T+E_{1} x_{1}+E_{2} x_{2}+E_{3} x_{3}\right]
\end{gathered}
$$


In the relations above, $x_{e}, x_{1}, x_{2}$ and $x_{3}$ represent the fraction of electrons, singly charged, doubly charged and triply charged ions in the vapor. The inclusion of these terms is needed to account for the ionization of the vapor that results from high temperature of the molecules being ejected and the absorption of laser energy. Furthermore, it is assumed that the electrons can be also treated as behaving like an ideal gas, following a Maxwell-Boltzmann distribution.

The energy source term in the Euler equations represents the absorption of laser energy by the plasma through the inverse Bremsstrahlung process and the emission of energy by the vapor through the Bremsstrahlung process. $\Phi$ represents the local laser irradiance and $\alpha_{I B}$ gives the local absorption coefficient, and the product of these two terms gives the local laser energy absorption. $\varepsilon_{\text {rad }}$ represents the radiation power loss, and assuming that the electrons have a Maxwellian velocity distribution, is ${ }^{19}$

$$
\varepsilon_{\text {rad }}=\left(\frac{2 \pi k T}{3 m_{e}}\right)^{1 / 2} \frac{32 \pi e^{6}}{3 h m_{e} c^{3}} n_{e} \sum_{z} z^{2} n_{z}
$$

where $e$ is the electron charge and $c$ is the velocity of light.

The absorption of laser radiation by the plasma occurs through electron-neutral and electron-ion inverse Bremsstrahlung processes. Electron-neutral absorption is important initially at lower temperatures but as the vapor temperature and consequently the number of charged particles increases, electron-ion absorption becomes dominant. The absorption coefficients for these two processes are

$$
\begin{gathered}
\alpha_{e-n}=\left[1-\exp \left(-\frac{h c}{\lambda k T}\right)\right] Q n_{e} n_{0} \\
\alpha_{e-i}=\left[1-\exp \left(-\frac{h c}{\lambda k T}\right)\right] \frac{4 e^{6} \lambda^{3} n_{e}}{3 h c^{4} m_{e}}\left(\frac{2 \pi}{3 m_{e} k T}\right)^{1 / 2} \sum_{z} z^{2} n_{z}
\end{gathered}
$$

where $\lambda$ is the laser wavelength, $Q$ is the cross section for photon absorption. The common factor in both formulas $(1-\exp (-h c / \lambda k T))$ accounts for stimulated emission ${ }^{1}$. The absorption coefficient $\alpha_{I B}$, was then approximated as the sum of these two absorption processes.

\begin{tabular}{ll}
\hline Specific Heat, $c$ & $940.0 \mathrm{~J} \mathrm{~kg}^{-1} \mathrm{~K}^{-1}$ (solid), $1289.0 \mathrm{~J} \mathrm{~kg}^{-1} \mathrm{~K}^{-1}$ (liquid) \\
Mass Density, $\rho$ & $2700.0 \mathrm{~kg} \mathrm{~m}^{-3}$ (solid), $2375.0 \mathrm{~kg} \mathrm{~m}^{-3}$ (liquid) \\
Melting Point, $T_{m}$ & $933.5 \mathrm{~K}$ \\
Heat of Evaporation, $H_{e v}$ & $10.8 \times 10^{6} \mathrm{~J} \mathrm{~kg}^{-1}$ \\
Atomic mass, $m$ & $27 \mathrm{~g} \mathrm{~mol}^{-1}$ \\
Absorption Coefficient, $\mu$ & $5.7 \times 10^{7} \mathrm{~m}^{-1}$ \\
Absorptivity, $A$ & 0.21 \\
First Ionization Potential, $E_{1}$ & $577.5 \mathrm{~kJ} \mathrm{~mol}^{-1}$ \\
Second Ionization Potential, $E_{2}$ & $1816.7 \mathrm{~kJ} \mathrm{~mol}^{-1}$ \\
Third Ionization Potential, $E_{3}$ & $2744.8 \mathrm{~kJ} \mathrm{~mol}^{-1}$ \\
\hline
\end{tabular}

Table 1. Parameter values in the model with aluminum as the target material.

\section{Results and Discussion}

Calculations were made for the $\mathrm{KrF}$ laser-aluminum interaction with material properties as given in Table 1 for fluences in the range of $1.0-2.0 \mathrm{~J} / \mathrm{cm}^{2}$. Aluminum was chosen as the target because it is a material of interest and to allow for comparison of the results with analytical models.

\section{A. Target Heating}

The aluminum target, initially at a temperature of $300 \mathrm{~K}$, is heated due to the absorption of the incident laser radiation. The temperature distribution in the target material for a fluence of $1.0 \mathrm{~J} / \mathrm{cm}^{2}$ is plotted for several representative times in Fig. 3. It appears that most of the laser energy is absorbed very close to the surface, and then is conducted into the material. The surface temperature for a fluences of 1.0 and $2.0 \mathrm{~J} / \mathrm{cm}^{2}$, as shown in Fig. 4 , is 
initially seen to increase, starts to drop as the amount of laser energy being absorbed decreases and the energy at the surface is conducted into the material. A maximum surface temperature of about $1850 \mathrm{~K}$ is obtained for a fluence of $2.0 \mathrm{~J} / \mathrm{cm}^{2}$ and about $1070 \mathrm{~K}$ for the $1.0 \mathrm{~J} / \mathrm{cm}^{2}$ case. The maximum surface temperature for each case occurs around $18 \mathrm{~ns}$, and it may be recalled that the laser beam has maximum intensity at $15 \mathrm{~ns}$. The results are qualitatively consistent with those of Ref. 10, although that study considered higher laser fluences.

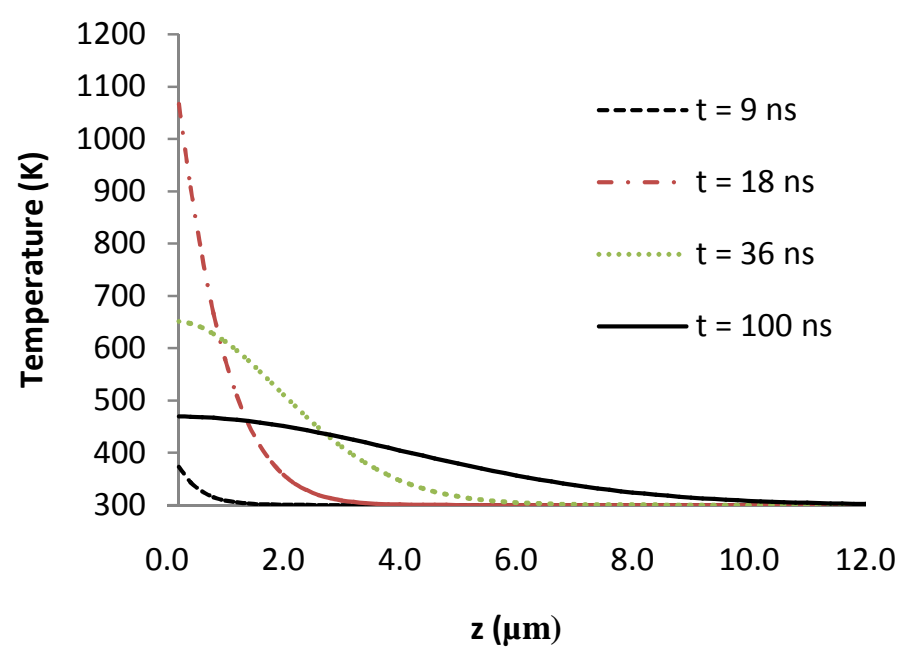

Figure 3. Temperature distribution in target.

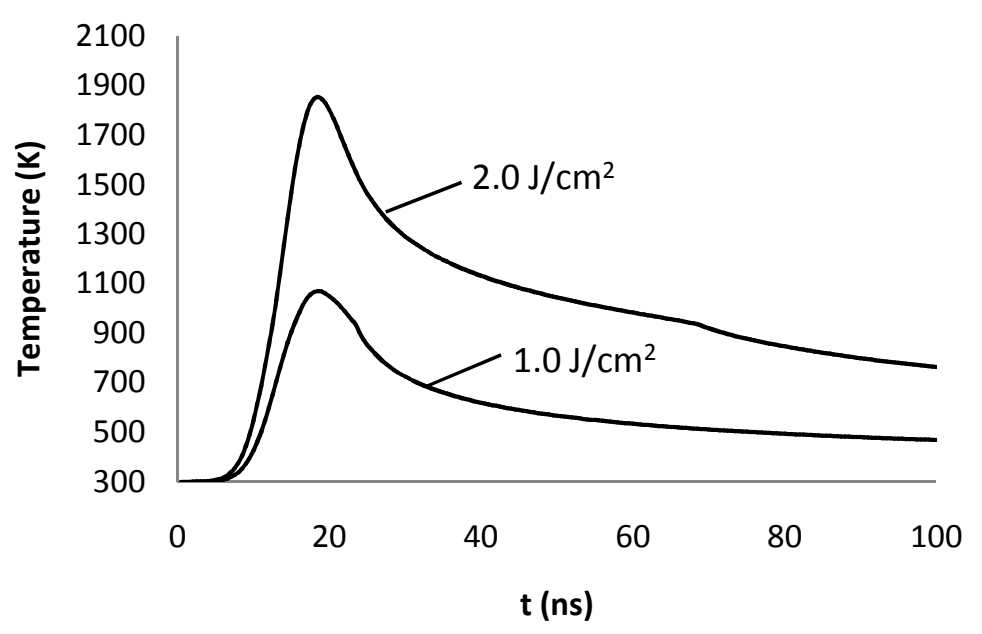

Figure 4. Surface temperature profiles.

\section{B. Ablation Rate}

The heating of the aluminum target will cause the target material to start evaporating, and the ablation rate generally increases as the surface temperature rises. The ablation rate of the aluminum target begins to increase sharply after about $15 \mathrm{~ns}$, and has a maximum value at around $18 \mathrm{~ns}$. The results also indicate that there is no significant material ejection after about $25 \mathrm{~ns}$. The results also show correlation with the target surface temperature rise. The ablation rate drops as the surface temperature begins to decrease. Material vaporization is also one of the factors causing the surface temperature to drop. The results for ablation rate indicate that a maximum ablation rate of $0.081 \mathrm{~kg} / \mathrm{m}^{2} \mathrm{~s}$ is obtained for a fluence of $2.0 \mathrm{~J} / \mathrm{cm}^{2}$. An ablation rate of $5.78 \times 10^{-8} \mathrm{~kg} / \mathrm{m}^{2} \mathrm{~s}$ is obtained for a fluence of $1.0 \mathrm{~J} / \mathrm{cm}^{2}$. The laser induced ablation of the aluminum target results in material loss from the surface. In an experimental setting, this would result in a crater around the laser beam incidence location. In this 1-D study, this material removal causes the thickness of the target material to be reduced. This shows most of the change in depth occurring between $13 \mathrm{~ns}$ and $25 \mathrm{~ns}$ after the beginning of the laser pulse irradiation. After that, as the ablation rate 
drops, the evaporation depth levels off. A fluence of 1.0 and $2.0 \mathrm{~J} / \mathrm{cm}^{2}$ produce an evaporation depth of about 0.021 and $0.105 \mathrm{~nm}$ respectively. The general trends obtained here are consistent with the analytical results of Ref. 10 and Ref. 12.

As mentioned above, there are two approaches to calculating the ablation rate. One is to assume that the plume expands to sound velocity at the edge of the Knudsen layer, and the other is to solve Eqs. (5) iteratively. Figures 5 and 6 show the difference in using these approaches to calculate the ablation rate and evaporation depths for a fluence of $1.8 \mathrm{~J} / \mathrm{cm}^{2}$. Using the sound speed assumption, the amount of material removed is about $1.44 \times 10^{14} \mathrm{~m}$ while the kinetic model solution results in $1.04 \times 10^{-14} \mathrm{~m}$ of material being removed. This significant difference is due to the plume not expanding to sound velocity at the edge of the kinetic layer, hence reducing ablation rate.

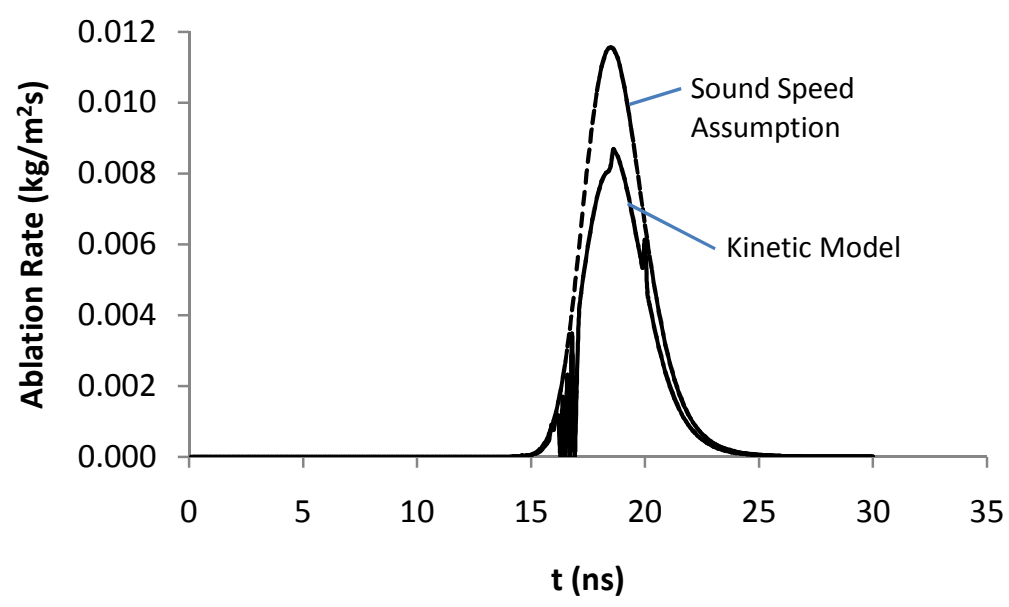

Figure 5. Ablation rate during and after laser pulse.

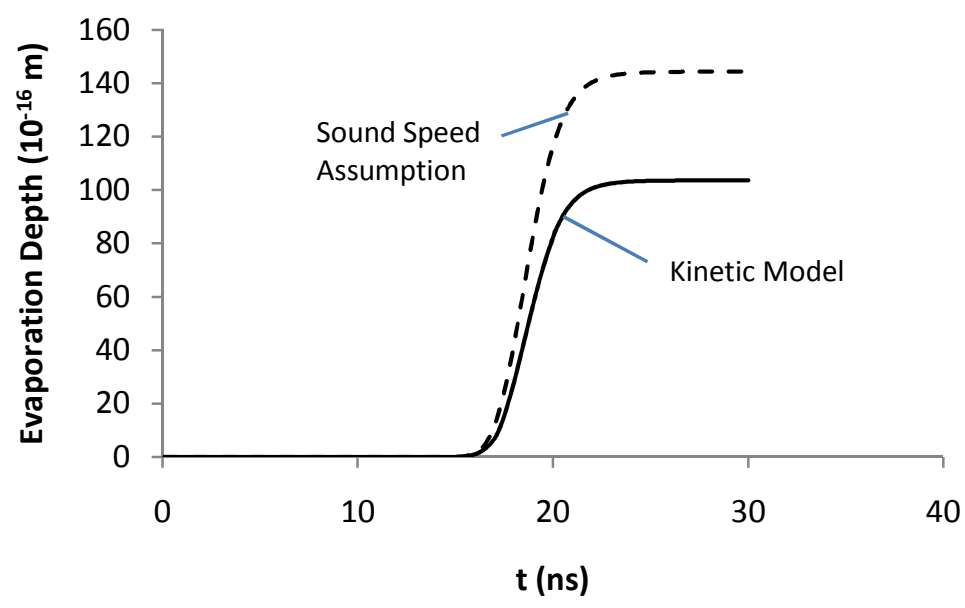

Figure 6. Evaporation depth of target material.

\section{Plasma Expansion}

The material ejected from the target forms a plume that expands into the vacuum surrounding the target. As long as the temperature and subsequently the ionization degree of the plume remain low, it is transparent to the laser beam passing through it, and energy absorption by the plasma is very small with almost all the laser energy reaching the aluminum target. At higher fluences, higher temperatures will be obtained in the plasma, and laser energy absorption becomes significant and thereby reduce the fraction of energy reaching the target. The results presented here are for cases where the effect of laser absorption on the plasma expansion is very small. 
In order to simulate a vacuum, the initial conditions assumed in the calculations are a vapor density of $10^{18} \mathrm{~m}^{-3}$ and a vapor temperature of $300 \mathrm{~K}$, corresponding to a residual pressure of less than $10^{-7} \mathrm{~atm}$. Furthermore, a zero vapor velocity and a residual ionization degree of $10^{-10}$ were assumed for the initial conditions. The boundary conditions at the edge of the Knudsen layer are obtained from Eqs. (5). The mass flow from the ablation drives the plume expansion.

Figures 7-9 shows the temperature, density and velocity profiles for several representative times during and after the laser pulse for the $2.0 \mathrm{~J} / \mathrm{cm}^{2}$ case. During the initial stage of plume expansion, the maximum temperature is obtained at the boundary. After $20 \mathrm{~ns}$, however, as the target surface temperature drops, the temperature at the edge of the Knudsen layer also drops. The front can be observed to move further into the vacuum as the plume becomes longer with time. The maximum temperature also decreases with time as the laser beam is terminated after $30 \mathrm{~ns}$ and the ablation rate drops. After about $25 \mathrm{~ns}$, the maximum temperature is observed near the plume front. For the 2.0 $\mathrm{J} / \mathrm{cm}^{2}$ fluence, a maximum temperature of around $1240 \mathrm{~K}$ was observed while a maximum temperature of about 715 $\mathrm{K}$ was observed for the $1.0 \mathrm{~J} / \mathrm{cm}^{2}$ case.

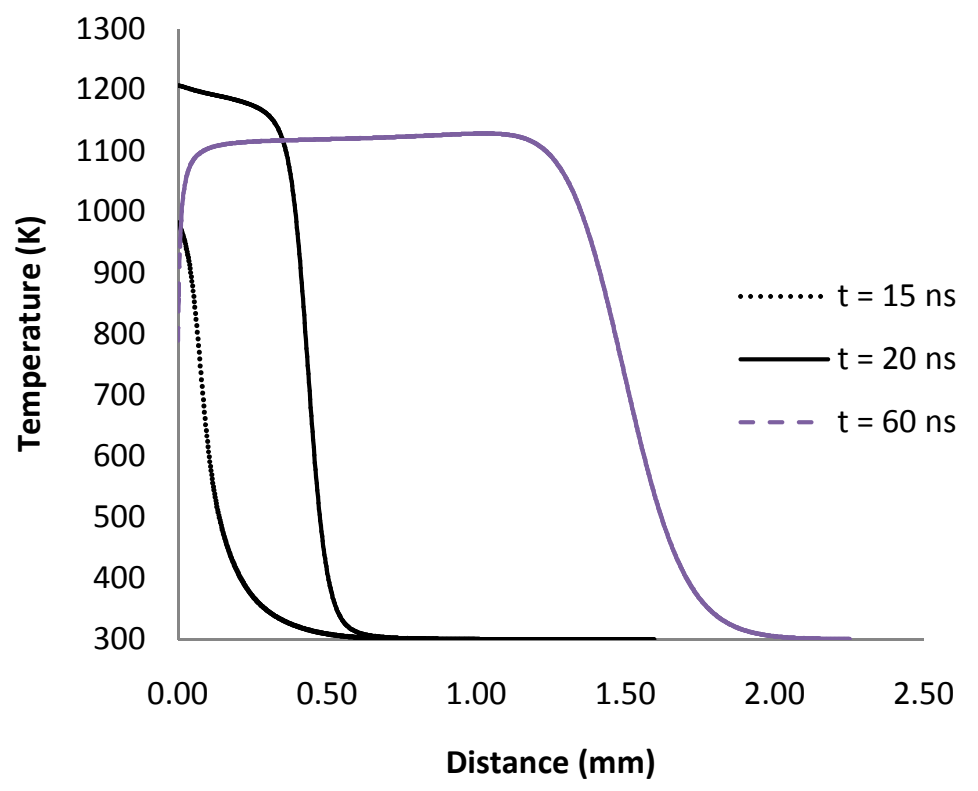

Figure 7. Temperature distribution in the plasma.

The vapor density variation behavior is shown in Figure 8. Initially, the density is maximum at the boundary and continues to rise as more and more material is ejected from the surface due to laser ablation. After about $20 \mathrm{ns,}$ as the amount of material being ablated drops, both the density at the boundary and the maximum density observed in the plume decreases. The location of maximum density moves into the plume as the plume expands. The plume itself becomes longer. For a fluence of $2.0 \mathrm{~J} / \mathrm{cm}^{2}$, the plume front has essentially moved to about $2.5 \mathrm{~mm}$ from the edge of the Knudsen layer after $100 \mathrm{~ns}$ with a maximum density of $2.5 \times 10^{19} \mathrm{~m}^{-3}$. A maximum density of about $2.0 \times 10^{21} \mathrm{~m}^{-3}$ is obtained after $18.5 \mathrm{~ns}$.

The vapor velocity variation with time shows behavior that is similar to the temperature profiles, as shown in Figure 9. The velocity at the edge of the Knudsen layer is assumed to be the sound velocity, which is a valid assumption when the ablated material expands into a vacuum. This assumption becomes invalid when the density and pressure of the surrounding conditions becomes large, but this situation is neglected in this study. The velocity is therefore maximum at the boundary during the early stages of plume expansion, but after about $20 \mathrm{~ns}$, the velocity is maximum close to the plume front, which is seen to move further into the vacuum. A maximum plume velocity of about $900 \mathrm{~m} / \mathrm{s}$ is obtained after about $50 \mathrm{~ns}$, at about $0.7 \mathrm{~mm}$ from the edge of the Knudsen layer. The results for the plasma expansion show a good qualitative agreement with Ref. 18 although that study used a copper target and a ruby laser. The general trends however are similar for the case below the plasma ignition threshold. In that study, the velocity and temperature profiles were similar as they are in this paper. 


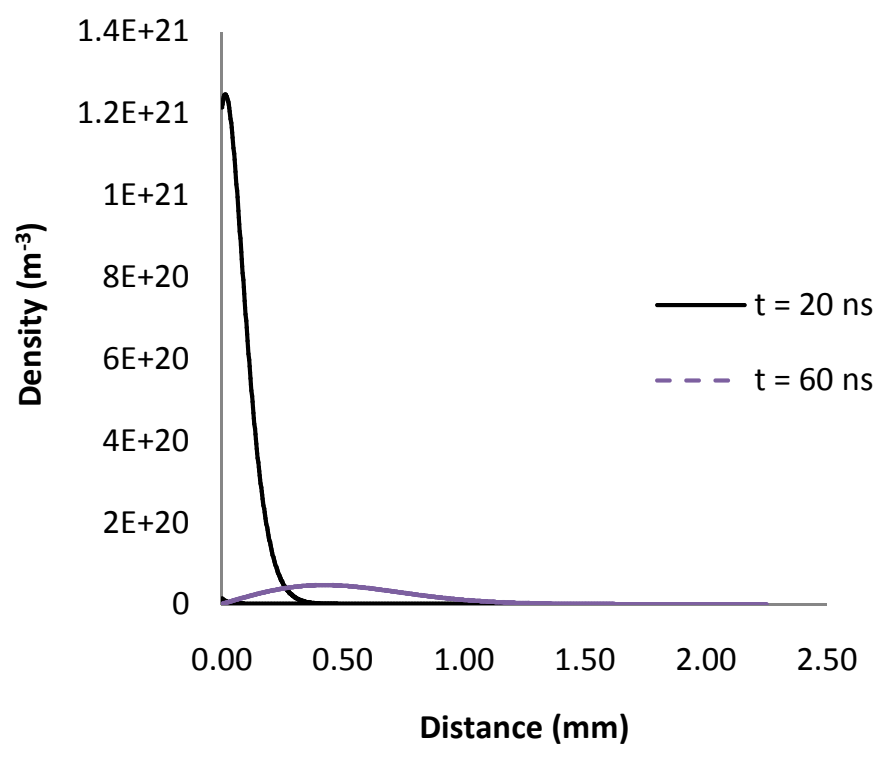

Figure 8. Density distribution in the plasma.

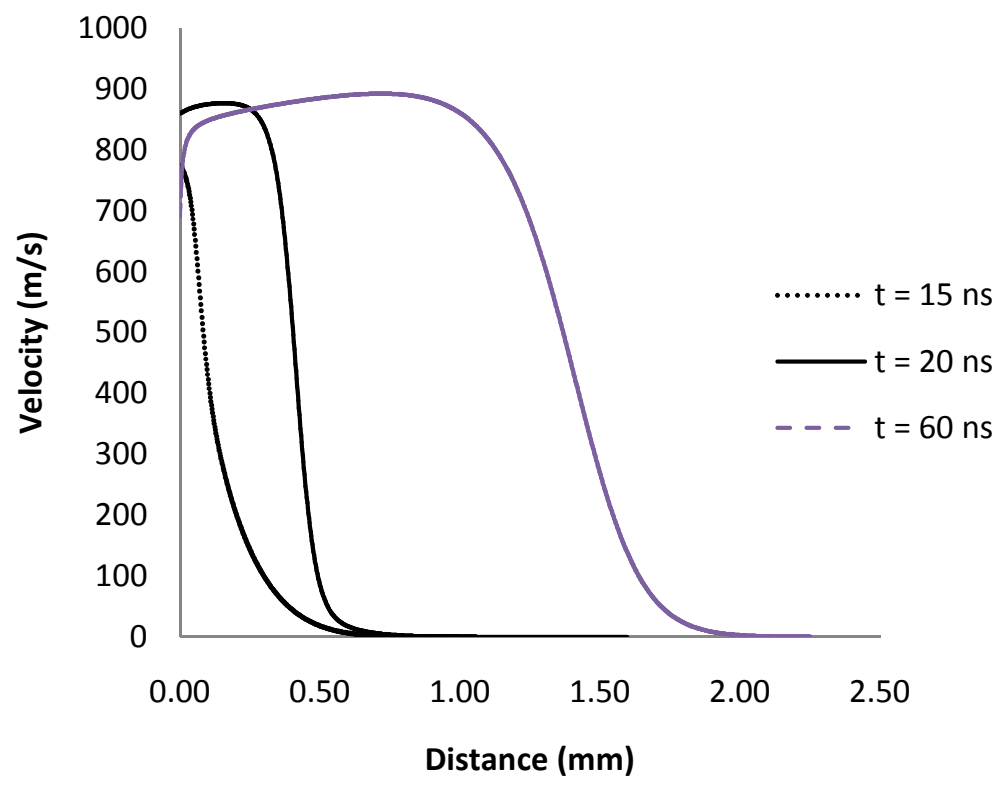

Figure 9. Velocity distribution in the plasma.

\section{Conclusion}

This paper has presented a model for the KrF laser-aluminum interaction for nanosecond pulsed laser ablation process in vacuum conditions. For the cases presented here, laser beam absorption by the plasma above the target is not significant - the plasma is generally transparent to the laser passing through it. This model describes the lasersolid interaction that results in target heating and vaporization leading to ablation, and the expansion of the plume formed in the vacuum due to the material being ejected from the target. The results include the temperature distribution in the aluminum target, the surface temperature profile, the ablation rate and the amount of evaporation, as well as the temperature, density and velocity profiles in the plasma plume. The results show acceptable agreement with results of other analytical ablation models, although the use of different laser beam profiles makes any accurate comparison difficult. 
The complexity of the problem involved makes it difficult to consider all the processes that takes place in lasersolid interaction. However, the inclusion of the important processes makes for relatively accurate predictions. For cases of higher fluences, when laser absorption by the plasma becomes significant, the complexity of the problem increases and requires further study.

\section{Acknowledgments}

The authors acknowledge the financial support of the Air Force Office of Scientific Research, Grant No. F4 9550-06-1-0393 (Dr Schmisseur, technical monitor). The authors also thank Drs. C. Jones and B. Mork for very useful discussions and suggestions.

\section{References}

${ }^{1}$ Radziemski, L.J. and Cremers, D.A., Laser-Induced Plasmas and Applications. Marcel Dekker, Inc., New York, 1989, Chaps. 1-2.

${ }^{2}$ Keidar, M., Boyd, I.D. and Beilis, I.I., "On the model of Teflon ablation in an ablation controlled discharge," J. Phys. D: Appl. Phys. Vol. 34, 2001, pp. 1675.

${ }^{3}$ von Allmen, M., Laser Beam Interactions with Materials. Springer Heidelberg, 1987.

${ }^{4}$ Semak, V.V. and Miller, T.F., "Modeling of Laser Charring and Material Removal in Fiberglass Materials," J. Directed Energy, Vol. 2, No. 1, 2006, pp. 5.

${ }^{5}$ Dushman, S., Vacuum Techniques. John Wiley \& Sons, Inc., 1949.

${ }^{6}$ Keidar, M., Fan, J., Boyd, I.D. and Beilis, I.I., "Vaporization of heated materials into discharge plasmas," J Appl. Phys., Vol. 89, No. 6, 2001, pp. 3095.

${ }^{7}$ Anisimov, S.I. Sov. Phys. JETP, Vol. 27, 1968, pp. 182.

${ }^{8}$ Peterlongo, A. , Miotello, A., and Kelly, R., "Laser-pulse sputtering of aluminum: Vaporization, boiling, superheating, and gas-dynamic effects," Phys. Rev. E, Vol. 50, No. 6, 1994, pp. 4716.

${ }^{9}$ Amoruso, S., "Modeling of UV pulsed-laser ablation of metallic targets," Appl. Phys. A, Vol. 69, 1999, pp. 323.

${ }^{10}$ Mele, A., Guidoni, A.G., Kelly, R., Flamini, C. and Orlando, S., "Laser ablation of metals: Analysis of surface-heating and plume-expansion experiments," Appl. Surf. Sci., Vol. 109, 1997, pp. 584.

${ }^{11}$ Yilbas, B.S., "Numerical approach to pulsed laser heating of Semi-Infinite Aluminum substance," Heat and Mass Trans. Vol. 31, 1996, pp. 279.

${ }^{12}$ Bogaerts, A., Chen, Z., Gijbels, R. and Vertes, A., "Laser ablation for analytical sampling what can we learn from modeling?," Spectrochimica Acta Part B, Vol. 58, 2003, pp. 1867.

${ }^{13}$ Keidar, M., Boyd, I.D., Luke, J. and Phipps, C., "Plasma generation and plume expansion for a transmission-mode microlaser ablation plasma thruster," J. Appl. Phys., Vol. 96, No. 1, 2004, pp. 49.

${ }^{14}$ Boardman, A.D., Cresswell, B. and Anderson, J.,, “An analytical model for the laser ablation of materials," Appl. Surf. Sci. Vol. 96-98, 1996, pp. 55.

${ }^{15}$ Leboeuf, J.N., Chen, K.R., Donato, J.M., Geohegan, D.B., Liu, C.L., Puretzky, A.A. and Wood, R.F., "Modeling of dynamical processes in laser ablation," Appl. Surf. Sci., Vol. 96-98, 1996, pp. 14

${ }^{16}$ Tannehill, J.C., Anderson, D.A. and Pletcher, R.H., Computational Fluid Mechanics and Heat Transfer. Taylor \& Francis, 1997.

${ }^{17}$ Burden, R.L., Faires, J.D., and Reynolds, A.C. (Eds.), Numerical Analysis, Prindle, Weber and Schmidt, Boston, 1978.

${ }^{18}$ Balazs, L., Gijbels, R., and Vertes, A. "Expansion of laser-generated plumes near the plasma ignition threshold," Anal. Chem, Vol. 63, 1991, pp. 314.

${ }^{19}$ Spitzer, L., Physics of Fully Ionized Gases, Interscience Publishers, London, 1956. 